\title{
РАДИАЦИОННЫЕ ИЗМЕНЕНИЯ ОПТИЧЕСКИХ И МЕХАНИЧЕСКИХ СВОЙСТВ МАТЕРИАЛОВ НА ОСНОВЕ $\mathrm{SiO}_{2}$
}

\author{
О.В. Никулина ${ }^{1}$, В.А. Степанов ${ }^{2,3}$ \\ ${ }^{1}$ Обнинское научно-производственное предприятие «Технология» им. А.Г. Ромашина, Калужская обл., \\ Киевское шоссе, д. 15, Обнинск, Российская Федерация, 249031 \\ E-mail: lunya23@yandex.ru \\ ${ }^{2}$ Обнинский институт атомной энергетики НИЯУ МИФИ, Калужская обл., Студгородок, д. 1, Обнинск, \\ Российская Федерация, 249030 \\ E-mail: stepanov@iate.obninsk.ru \\ ${ }^{3}$ ФГБУН Межведомственный центр аналитических исследований в области физики, химии и биологии \\ при Президиуме РАН, ул. Профсоюзная, д. 65, с. 6, Москва, Российская Федерация, 117997 \\ Показано, что радиационные изменения механических и оптических свойств квар- \\ цевых стекол имеют одинаковую природу и связаны с разделением электрического заряда \\ в нано-неоднородном объеме стекол. Разделение электрического заряда в стеклах проис- \\ ходит до поглощенных доз порядка 1 МГр. Локальное разделение электрического заряда \\ вследствие появления кулоновских сил приводит к радиационному кулоновскому упрочне- \\ нию стекол и композитов на их основе.
}

Ключевые слова: кварцевые стекла, гамма-облучение, оптические свойства, радиационное упрочнение

\section{RADIATION CHANGES IN THE OPTICAL AND MECHANICAL PROPERTIES OF MATERIALS BASED ON $\mathrm{SiO}_{2}$}

\begin{abstract}
O.V. Nikulina ${ }^{1}$, V.A. Stepanov $v^{2,3}$
${ }^{1}$ JSC «ORPE «Technologiya» named after A.G. Romashin, Kaluga, Kievskoye Shosse, 15, Obninsk, Russian Federation, 249031

E-mail: lunya23@yandex.ru

${ }^{2}$ National research nuclear university (NRNU) mephi obninsk institute for nuclear power engineering (OINPE), Studgorodok, Kaluga region, 1, Obninsk, Russian Federation, 249040

E-mail: stepanov@iate.obninsk.ru

${ }^{3}$ Federal State State-financed Institution of Sciences Interdepartmental Center of Analytic Studies in the Sphere of Physics, Chemistry and Biology under Presidium of Russian Academy of Sciences (ICAS RAS), Trade Union Street, 65, p. 6, Moscow, Russian Federation, 117342

It is shown that the radiative changes in the mechanical and optical properties of silica glasses are of the same nature and are associated with the separation of the electric charge in the nano-inhomogeneous glass volume. The separation of electric charge in glasses occurs up to absorbed doses of the order of 1 MGy. Local separation of the electric charge due to the appearance of Coulomb forces leads to radiation Coulomb hardening of glasses and composites with glasses.
\end{abstract}

Key words: quartz glass, gamma irradiation, optical properties, radiation hardening

Для цитирования:

Никулина О.В., Степанов В.А. Радиационные изменения оптических и механических свойств материалов на основе $\mathrm{SiO}_{2}$. Рос. хим. ж. (Ж. Рос. хим. об-ва). 2021. Т. LXV. № 3. С. 51-56

For citation:

Nikulina O.V., Stepanov V.A. Radiation changes in the optical and mechanical properties of materials based on $\mathrm{SiO}_{2}$. Ros. Khim. Zh. 2021. V. 65. N 3. P. 51-56 


\section{ВВЕДЕНИЕ}

Кварцевое стекло относится к числу наиболее широко применяемых на практике оптических материалов. Оно характеризуется высоким коэффициентом оптического пропускания в УФ, видимой и ближней ИК областях спектра, является основой для получения оптических волокон, которые применяются в системах связи и в измерительных приборах. Высокие механическая прочность и термостойкость, устойчивость к действию многих кислот и воды позволяют применять кварцевые стекла в качестве элементов конструкционных материалов. В виде волокон или стеклотканей оно входит в состав различных композиционных материалов, из которых создаются элементы конструкций в авиационной отрасли, в транспорте и строительстве.

Кварцевое стекло относится к числу диэлектрических материалов с повышенной радиационной стойкостью, поэтому, как в качестве оптических элементов, так и в качестве компонент конструкционных композитов, оно применяется и в атомной технике, и в авиакосмических системах. Тем не менее, с дозой облучения свойства кварцевых стекол и материалов на их основе меняются. Происходит ухудшение оптических свойств и, как правило, возрастает механическая хрупкость. Понимания механизмов радиационно-индуцированных процессов в кварцевых стеклах к настоящему времени недостаточно для направленного в связи с условиями эксплуатации выбора типов стекол, выбора условий их получения, примесного состава. На практике такой выбор осуществляется в результате экспериментального перебора или интуитивно.

Трудность развития радиационного материаловедения кварцевых стекол и стекол, вообще, связана с тем, что развитые и используемые для кристаллических материалов подходы, основанные на изучении механизма влияния образующихся при облучении точечных, линейных и других дефектов периодической структуры, для стекол не применимы. Кристаллических дефектов, если их пытаться определить в неупорядоченной структуре, в исходном стекле больше, чем может образоваться в условиях радиации.

Известен иной подход в исследованиях радиационно-индуцированных процессов, учитывающий реальную нано-неоднородную структуру стекла $[1,2]$. На примере фторидных стекол были получены результаты, согласно которым изменения оптических свойств нужно связывать не с радиационными дефектами, а с радиационно-индуцированным разделением электрического заряда в присущей всем стеклам нано-неоднородной структуре. Такой подход представляется универсальным в радиационном материаловедении диэлектриков, и его можно было бы применять для разных типов стекол. Для проверки общности он должен быть исследован при описании не только оптических радиационных изменений, но и других свойств стекол. В настоящей работе мы исследовали радиационные изменения как оптических, так и механических свойств кварцевых стекол с учетом их реальной нано-структуры.

\section{СТРУКТУРА И ОПТИЧЕСКИЕ СВОЙСТВА ОБЛУЧЕННЫХ СТЕКОЛ}

При радиационных воздействиях происходит уменьшение спектрального диапазона прозрачности стекол. Например, спектральный диапазон прозрачности кварцевых стекол в результате радиационного облучения может сужаться от 0,2-4,0 мкм до 0,3-2 мкм [3]. Фторидные стекла прозрачны в более широком спектральном диапазоне от 0,2-7,0 мкм, который после облучения уменьшается до 0,4-6,0 мкм [1]. При этом для всех типов стекол, вне зависимости от их химического состава, в коротковолновой части спектра после облучения происходит размытие и сдвиг фундаментального края поглощения в область больших длин волн.

На рис. 1 представлены спектры оптического поглощения кварцевых стекол КУ-1 и КИ до и после облучения на гамма-источнике $\mathrm{Co}^{60}$. Кварцевые стекла КУ-1 содержат минимальное количество металлических примесей $\mathrm{Al}, \mathrm{Fe}, \mathrm{Na}$, менее 0,001\%. В стеклах КИ, напротив, содержание металлических примесей $\mathrm{Zr}, \mathrm{Sr}, \mathrm{As}$, Мо и Ва достигает $1 \%$. Поэтому исходный край поглощения стекла КИ сдвинут в красную область.

После облучения происходят размытие и сдвиг фундаментального края поглощения в область больших длин волн. Такие изменения в спектрах оптического поглощения стекол невозможно объяснить появлением при облучении дефектов структуры - центров оптических переходов. В этом случае приходилось бы полагать, что при облучении вне зависимости от типа стекла образуется непрерывный ряд центров окраски со сплошным спектром энергетических уровней, при котором оптические переходы становятся возможными во всем видимом и УФ диапазонах. В работах $[1,2]$ показано, что одинаковый характер радиационных изменений оптических свойств стекол различного состава обусловлен общим, присущим для всех стекол, характером их наноструктуры.

Еще в 1921 г. А.А. Лебедевым было высказано предположение, что силикатное стекло имеет 
скрытно-кристаллическое строение. Структуру стекол можно рассматривать как неоднородную среду из микроструктурных образований, каждое из которых характеризуется упорядоченным расположением атомов. Микро-неоднородность стекол подтверждена с использованием различных методов исследования [4]. Структурно-упорядоченные области (домены), размер которых определяется корреляционным радиусом ближнего порядка структуры, могут варьироваться в различных стеклах в пределах $d=1-10$ нм. Между доменами могут располагаться области такого же размера с разупорядоченной структурой $[5,6]$.

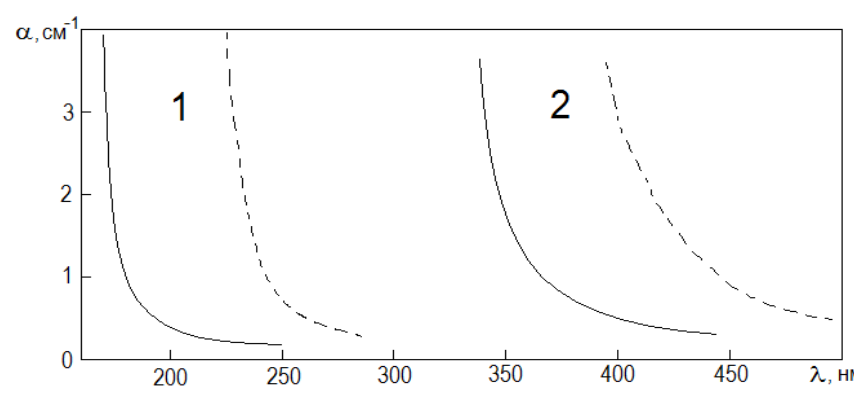

Рис. 1. Спектры оптического поглощения силикатных стекол до (сплошная линия) и после гамма облучения (пунктирная линия) 1 - КУ-1 (доза $10^{8}$ Гр), 2 - КИ (доза $4 \cdot 10^{5}$ Гр)

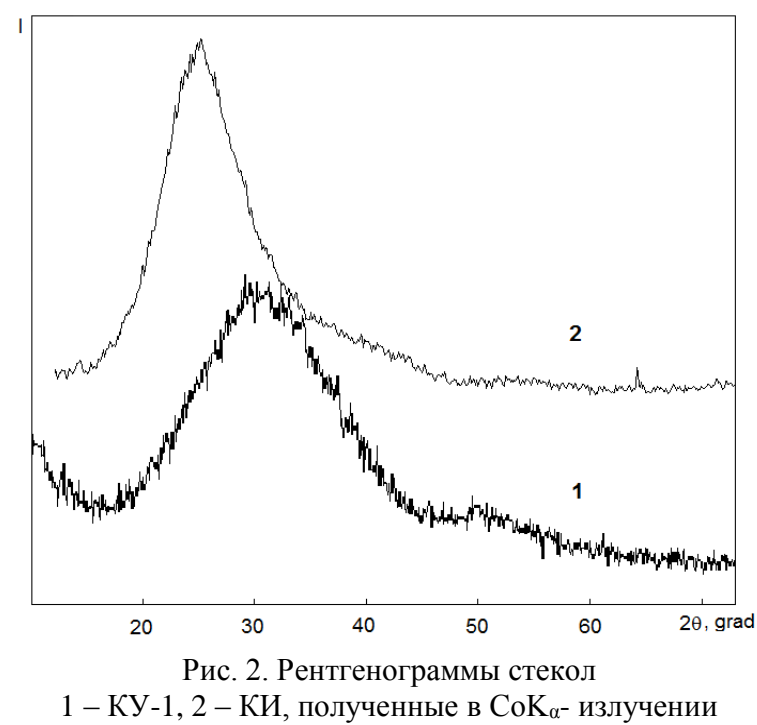

Доменное нано-структурное строение стекол КУ-1 и КИ можно определить с помощью классического рентгеноструктурного анализа. На рис. 2 показаны рентгенограммы стекол, полученные в режиме пошагового сканирования (шаг съемки 0,1 , время набора импульсов в точке $6-10$ c). В качестве экспрессного и оценочного метода определения размера областей когерентного рассеяния (ОКР) использовали программу РНAN\% (НИТУ
«МИСиС») в режиме метода подгонки спектра, аппроксимацией экспериментального спектра линейной комбинацией теоретических спектров фаз и фона с автоматической оптимизацией периодов решетки и параметров уширения линий фаз, диаметра блока и среднеквадратичной микродеформации. При подгонке минимизируется функция, являющаяся суммой квадратов разностей экспериментального и модельного спектров в каждой точке съемки. Модельный спектр является суммой модельных спектров отдельных фаз, вклад от каждой фазы пропорционален ее объемной доле. При расчете модельного спектра от каждой фазы учитывается вклад блочного и деформационного уширения. Форма линий описывается лоренцианом соответствующей кратности. Получено, что при использовании в качестве базовой фазу кристобалита размер доменов $d$ стекла КУ-1 составляет $1,00 \pm 0,01$ нм, а стекла КИ - 1,32 $\pm 0,01$ нм.

Ионизирующее облучение приводит к разделению электрического заряда вследствие перезарядки электрон-дырочных ловушек и к возникновению оптических неоднородностей в объеме стекла из-за поляризации объема вблизи заряженных центров [2]. Радиационные носители тока (электроны и дырки) мигрируют к структурным неоднородностям, к границам доменов. Это приводит к возникновению разделенных областей когерентного рассеяния света размером $d$. Анализ спектров поглощения позволил заключить, что спектральный ход оптических потерь в радиационно-окрашенных стеклах наиболее хорошо соответствует спектральному ходу потерь на рассеяние в оптически неоднородной среде $[1,2]$. Поскольку в видимом диапазоне длина волны света $\lambda>30 d$, то оптические потери за счет рассеяния и спектральный ход коэффициента поглощения (потерь) можно описывать с помощью теории рассеяния Релея [7]:

$$
\sigma=\frac{4 p r_{e}^{2}}{3} \cdot \frac{\left(n_{e} d^{3}\right)^{2}}{\left(\left(\frac{\lambda}{\lambda_{0}}\right)^{2}-1\right)^{2}} \cdot n_{d}
$$

где $r_{e}$ - классический радиус электрона $\left(2,8 \cdot 10^{-15} \mathrm{M}\right)$, $\lambda_{0}$ - длина волны, соответствующая ширине запрещенной зоны, $n_{e}-$ концентрация валентных электронов, по порядку величины атомная концентрация, $n_{d}-$ концентрация активных в рассеянии доменов. Если положить, что вся поглощенная энергия гамма-излучения расходуется на рождение носителей заряда, то с увеличением поглощенной дозы $D$ в отсутствии электрон-дырочной аннигиляции, когда все рожденные свободные электроны захватываются на ловушки, концентрация активных в рассеянии доменов растет как: 


$$
n_{d}=\frac{D \rho}{E_{g}} \cdot \frac{1}{6 d^{2} n_{e}^{\frac{2}{3}}}
$$

где $\rho$ - плотность стекла. Подставляя (2) в (1), получим зависимость от дозы:

$$
\alpha=\frac{2 \pi r_{e}^{2}}{9} \cdot \frac{D \rho}{E_{g}} \cdot\left(n_{e}\right)^{\frac{4}{3}} \cdot \frac{1}{\left(\left(\frac{\lambda}{\lambda_{0}}\right)^{2}-1\right)^{2}} \cdot d^{4}
$$

Размер рассеивающих свет доменов в соответствии с таким механизмом радиационно-наведенных оптических потерь в стеклах определяется корреляционным радиусом ближнего порядка, который к тому же соответствует размеру мельчайших элементов структуры, размеру областей когерентного рассеяния рентгеновского излучения. Вычисленные из спектров оптических спектров облученных стекол (рис. 1) с помощью формулы (3) значения размеров областей когерентного рассеяния света при 300-600 нм для КУ-1 - 1,1 нм, хорошо соответствовали значениям размеров когерентного рассеяния (структурных блоков), вычисленных из спектров рентгеновской дифракции: КУ-1 - 1,0 нм, Расхождение между оптическими и рентгеновскими значениями имеют место, когда в стеклах присутствуют глубокие центры захвата электронов, например, примеси ионов переходных металлов. Например, для стекла КИ вычисленное из оптических спектров значение 8,1 нм значительно превышает значение области когерентного рентгеновского рассеяния 1,3 нм. В соответствии с (3) оптические потери в облученном стекле сильно зависят от размера доменов, пропорционально $\mathrm{d}^{4}$.

Можно оценить дозу насыщения, при которой весь объем стекла разбивается на области оптического рассеяния:

$$
D_{d}=\frac{3 E_{g}}{d \rho} \cdot\left(n_{e}\right)^{\frac{2}{3}}
$$

Это выражение для кварцеых стекол дает оценку $D_{d} \sim(0,7-2) \cdot 10^{6} \quad \Gamma \mathrm{p}$, которая вполне согласуется с экспериментальными значениями доз насыщения радиационной окраски [3].

\section{ИНДУЦИРОВАННОЕ ОБЛУЧЕНИЕМ КУЛОНОВСКОЕ УПРОЧНЕНИЕ СТЕКОЛ}

Исследования [8-10], проведенные на керамических диэлектрических материалах, указывают на эффективное радиационно-индуцированное образование разноименно электрически заряженных микрообластей в объеме. Было обнаружено, что при протонном облучении (энергия 8 МэВ, мощность дозы $\left.1,4 \cdot 10^{4} \Gamma \mathrm{p} / \mathrm{c}\right)$ величина избыточного положительного заряда в зернах 1-10 мкм керамики $\mathrm{Al}_{2} \mathrm{O}_{3}: \mathrm{Cr}^{3+}\left(10^{-2}\right.$ масс. \% хрома) превышает $10^{-5} \mathrm{Kл} / \mathrm{cm}^{3}$, а созданная этим зарядом напряженность электрического поля более $10^{4} \mathrm{~B} / \mathrm{cm}$. Такие значения были рассчитаны по увеличению интенсивности радиолюминесценции полосы ионов $\mathrm{Cr}^{3+}$ (690 нм). Механизм радиолюминесценции обусловлен захватом электронов ионами $\mathrm{Cr}^{4+}$ и высвечиванием образующихся ионов $\mathrm{Cr}^{3+}$. В керамике происходит сток электронов на границы, что приводит к избыточному положительному заряду и повышению концентрации ионов $\mathrm{Cr}^{4+}$ в зерне. Интенсивность этой полосы в монокристаллах $\mathrm{Al}_{2} \mathrm{O}_{3}: \mathrm{Cr}^{3+}$ оставалась в этих же условиях постоянной. Оценка плотности электрического заряда при протонном облучении на границах зерен керамики $\mathrm{Al}_{2} \mathrm{O}_{3}: \mathrm{Cr}^{3+}$ дает $3 \cdot\left(10^{-6}\right.$ $\left.10^{-5}\right)$ Кл/ $\mathrm{m}^{2}$. Это соответствует перезарядке до $10 \%$ от всего количества ионов хрома.

В кварцевых стеклах рост интенсивности радиолюминесценции с дозой облучения также связывался с радиационно-индуцированным разделением заряда [11]. Локальное разделение электрического заряда вследствие появления кулоновских сил приводит к радиационному упрочнению структурно-неоднородных диэлектрических материалов. Оценим кулоновское упрочнение в стекле. Элементы структуры неоднородного распределения заряда в объеме облученного стекла представим плоскими заряженными конденсаторами размером $d$ с плотностью заряда на обкладках $\rho_{s}$, которая пропорциональна плотности заряженных ловушек для электронов и дырок на границах доменов. Электрическая энергия плоского конденсатора $U=\frac{Q^{2}}{2 C}$, где $C=\varepsilon \varepsilon_{0} S / d, S$ - площадь обкладок, $d$ - расстояние между ними. Между обкладками возникает давление из-за кулоновского притяжения $\sigma=-\frac{1}{S} \frac{\partial U}{\partial d}$, и если $Q=\rho_{S} S$, то

$$
\sigma=-\frac{\rho_{s}^{2}}{2 \varepsilon \varepsilon_{0}}
$$

Знак минус означает сжатие.

В керамике $\mathrm{Al}_{2} \mathrm{O}_{3}: \mathrm{Cr}^{3+}$ при протонном ионизирующем облучении заряжается до $10^{-1}$ электрон-дырочных связанных с $\mathrm{Cr}^{3+}$ ловушек. Если положить, что в кварцевых стеклах при облучении на границах доменов максимальный процент заряженных ловушек тот же, а их количество порядка концентрации $\mathrm{Si}-\mathrm{O}$ связей, то для кулоновского упрочнения получаем верхнюю оценку $10^{8}$ Па. Это значение, однако, на порядок превышает предел текучести и для некоторых стекол, даже предел прочности. При размере доменов $d=1$ нм (например, в стеклах КУ-1) в поверхностном слое такой же толщины располагается до 30 формульных единиц $\mathrm{SiO}_{2}$. Это дает нижнюю оценку количества заряженных ловушек около 3\% и нижнюю оценку упрочнения до $10^{7}$ Па, которая, по-видимому, близка к реальным значениям. 


\section{РАДИАЦИОННОЕ УПРОЧНЕНИЕ СИЛИКАТНЫХ КОМПОЗИТОВ}

Оценка кулоновского упрочнения в облученных силикатных стеклах дает значения порядка десятков МПа при поглощенных дозах, близких к дозе насыщения (4) до 1 МГр. Такое упрочнение приводит к увеличению прочности материалов на основе стекол $\mathrm{SiO}_{2}$. В ОА «ОНПП «Технология» им. А.Г.Ромашина» методами вакуумного и контактного формования производится такой неорганический композиционный материал аэрокосмического назначения марки ХАФСкв [12]. В качестве текстурированного наполнителя в нем используется ткань из аморфного кварцевого волокна с содержанием примесей не более $10^{-2}$. Неорганическое связующее на основе фосфатов алюминия по- лучают из водных растворов кислых фосфорнокислых солей алюминия и хрома, в которые добавляют различные аппреты и микрошлифпорошки из электрокорунда и аэросила для химической и механической защиты наполнителя. Такой композит пригоден для работы вплоть до $1500{ }^{\circ} \mathrm{C}$.

Прочность композита определяется прочностью кварцевого наполнителя. Увеличение прочности от 10 МПа можно обнаружить в механических испытаниях материалов даже с учетом неизбежного разброса результатов измерений. Для проведения испытаний было изготовлено 30 образцов ХАФСкв размерами $(20 \times 10 \times 2)$ мм, половина из которых была облучена на гамма-источнике $\mathrm{Co}^{60}$. Механические испытания материала проводились по ГОСТ 4651-82 на установке LFM-50.

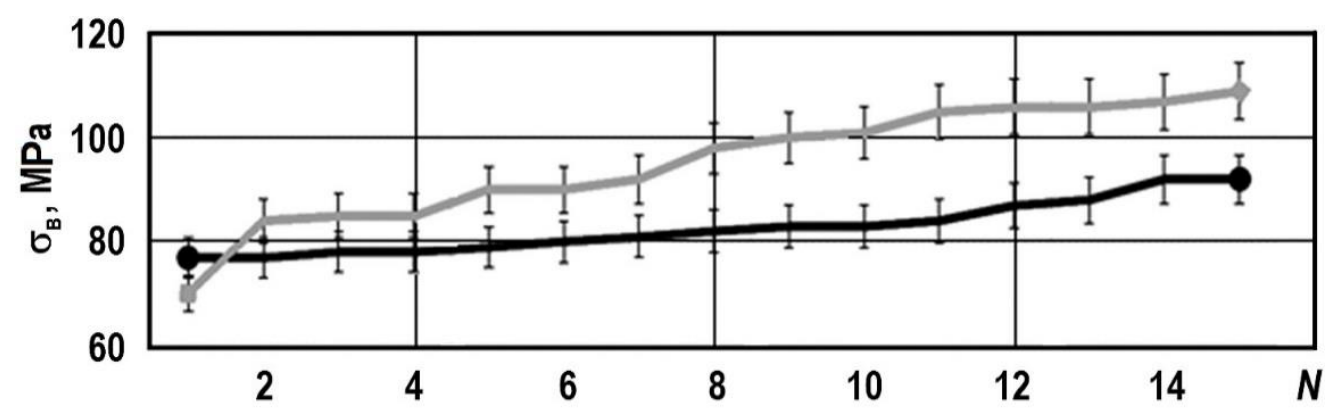

Рис. 3. Предел прочности при изгибе образцов материала ХАФСкв до (черная кривая) и после гамма-облучения с дозой $1,34 \cdot 10^{5} \Gamma \mathrm{p}$ (серая кривая)

Результаты механических испытаний приведены на рис. 3. После гамма-облучения композита не происходит заметных изменений модуля упругости до $3 \%$. Предел прочности гамма-облученного композита превышает значение исходного материала. При этом максимальное наблюдаемое упрочнение 20 МПа находится в интервале приведенных оценок кулоновского упрочнения кварцевого стекла.

\section{ВЫВОДЫ}

Радиационные изменения механических и оптических свойств кварцевых стекол имеют одинаковую природу и связаны с радиационно-индуцированным разделением электрического заряда

$$
\text { ЛИТ ЕР А Т У Р А }
$$

1. Stepanov V.A., Baskov P.B., Chernov V.M., Fedorov V.D., Khorozova O.D., Kurdyavko P. V., Sakharov V. V., Stepanov P.A. Fluoride glasses as materials for radiation optics. Proc. of XIII ${ }^{\text {th }}$ Int. Symposium on Non-Oxide Glasses and New Optical Glasses, September 9 -13, 2002 Pardubice, Czech Republic, Part II, P. 674-677.

2. Степанов B.A. Радиационно-индуцированные изменения оптических свойств стекол. Тез.докл. 7 Межд. Конф. на присущих стеклам структурных нано-неоднородностях. Локальное разделение электрического заряда происходит до поглощенных доз порядка 1 МГр и приводит к радиационному упрочнению стекол вследствие появления кулоновских сил. Кулоновское упрочнение кварцевых стекол может составлять $\sim 10^{7}$ Па.

Испытания на механическую прочность композиционного материала с наполнителем из кварцевого стекла подтвердили сделанные оценки. После гамма-облучения $1,34 \cdot 10^{5}$ Гр предел прочности композита ХАФСкв на основе ткани из силикатных волокон увеличивался на величину до 20 МПа, что соответствует значению кулоновского упрочнения.

\section{REFERENCES}

1. Stepanov V.A., Baskov P.B., Chernov V.M., Fedorov V.D., Khorozova O.D., Kurdyavko P.V., Sakharov V.V., Stepanov P.A. Fluoride glasses as materials for radiation optics. Proc. of XIII ${ }^{\text {th }}$ Int. Symposium on Non-Oxide Glasses and New Optical Glasses, September 9 -13, 2002 Pardubice, Czech Republic, Part II. P. 674-677.

2. Stepanov V.A. Radiation-induced changes in the optical properties of glasses. Proc. of 7 Int. Conference. Inzhenernyye 
Инженерные проблемы термоядерных реакторов., С.Петербург, 28-31 октября 2002 г., С. 195.

3. Арбузов В.И. Основы радиационного оптического материаловедения - СПб.: СПб ГУ ИТМО, 2008. 284 с.

4. Малиновский В.К., Суровцев Н.В. Неоднородность в нанометровом масштабе как универсальное свойство стекол. Физ. хим. стекла. 2000. Т. 26. № 3. С. 315-321.

5. Malinovsky V.K., Noviokov V.N. The nature of the glass transition and the excess low-energy density of vibrational states in glasses. J. Phys.: Condens. Matter. 1992. V. 4. P. L139.

6. Малиновский В.К, Новиков В.Н., Суворовцев Н.В., Шебанин А.П. Изучение аморфных состояний $\mathrm{SiO}_{2}$ методом комбинационного рассеяния. ФТТ. 2000. Т. 42. Вып. 1. С. 62-68.

7. Фабелинский И. Л. Молекулярное рассеяние света. М.: Высшая школа. 1965. 512 с.

8. Chernov V.M., Khorasanov G.L., Plaksin O.A., Stepanov V.A., Stepanov P.A., Belyakov V.A. Electrical and Optical Characteristics of Dielectrics for Fusion Use under Irradiation. Journal of Nuclear Materials. 1998. V. 253. P. 175.

9. Plaksin O.A., Chernov V.M., Stepanov P.A., Stepanov V.A. Radiation-Induced Electrical and Optical Processes in Materials Based on $\mathrm{Al}_{2} \mathrm{O}_{3}$. Journal of Nuclear Materials. 1999. V. 271-272. P. 496.

10. Плаксин О.А., Степанов В.А. Радиационно-индуцированные электрические и оптические процессы в материалах на основе $\mathrm{Al}_{2} \mathrm{O}_{3}$. Оптика и спектроскопия. 2001. Т. 90. №4. C. $612-621$.

11. Деменков П.В., Плаксин О.А., Степанов В.А., Степанов П.А., Чернов В.М. Кинетика люминесценции кварцевых стекол при облучении протонами. Письма в ЖТФ. 2000. Т. 26. Вып. 11. С. 57-61.

12. Степанов П.А., Атрощенко И.Г., Стародубцева Н.И., Шуткина О.В., Мельников Д.А. Разработка высокотемпературных композиционных материалов теплозащитного и радиотехнического назначения. Перспективные материалы. 2014. №10. С. 17-21. problemy termoyadernykh reaktorov [Engineering Problems of Fusion Reactors]. St. Petersburg, 28-31 October. 2002. P. 195.

3. Arbuzov V.I. Fundamentals of Radiation Optical Materials Science. St. Petersburg, ITMO University Publ., 2008, 284 p.

4. Malinovsky, V.K., Surovtsev, N.V. Inhomogeneity on the nanometer scale as a universal property of glasses. Glass Physics and Chemistry. 2000. V. 26. P. 217-222.

5. Malinovsky V.K., Noviokov V.N. The nature of the glass transition and the excess low-energy density of vibrational states in glasses. J. Phys.: Condens. Matter. 1992. V. 4. P. L139.

6. Malinovsky V.K, Novikov V.N., Suvorovtsev N.V., Shebanin A.P. Investigation of amorphous states of $\mathrm{SIO}_{2}$ by Raman scattering spectroscopy. Physics of the solid state. 2000. V. 42. N 1. P. 65-71.

7. Fabelinsky I.L., Molecular scattering of light. Moscow, Vysshaya shkola Publ. 1965. 512 p.

8. Chernov V.M., Khorasanov G.L., Plaksin O.A., Stepanov V.A., Stepanov P.A., Belyakov V.A. Electrical and Optical Characteristics of Dielectrics for Fusion Use under Irradiation. Journal of Nuclear Materials. 1998. V. 253. P. 175.

9. Plaksin O.A., Chernov V.M., Stepanov P.A., Stepanov V.A. Radiation-Induced Electrical and Optical Processes in Materials Based on $\mathrm{Al}_{2} \mathrm{O}_{3}$. Journal of Nuclear Materials. 1999. V. 271-272. P. 496.

10. Plaksin O.A. and Stepanov V.A. Radiation-Induced Electrical and Optical Processes in the Materials Based on $\mathrm{Al}_{2} \mathrm{O}_{3}$. Optics and Spectroscopy. 2001. V. 90. N 4. P. 542-551.

11. Demenkov P.V., Plaksin O.A., Stepanov V.A., Stepanov P.A., and Chernov V.M. Luminescence Kinetics in ProtonIrradiated Quartz Glasses. Technical Physics Letters. 2000. V. 26. P. 475-477.

12. Stepanov P.A., Atroshchenko I.G., Starodubtseva N.I., Shutkina O.V., Melnikov D.A. Development of high-temperature composite materials for heat-shielding and radio engineering purposes. - Perspektivnyye materialy. 2014. N 10. P. 17-21.

Поступила в редакииюю (Received) 01.08.2021

Принята к опубликованию (Accepted) 03.09.2021 